\title{
A millisecond quantum memory for scalable quantum networks
}

\author{
Bo Zhao ${ }^{1 \star}$, Yu-Ao Chen ${ }^{1,2 \star \dagger}$, Xiao-Hui Bao ${ }^{1,2}$, Thorsten Strassel ${ }^{1}$, Chih-Sung Chuu ${ }^{1}$, Xian-Min Jin ${ }^{2}$, \\ Jörg Schmiedmayer ${ }^{3}$, Zhen-Sheng Yuan ${ }^{1,2}$, Shuai Chen ${ }^{1}$ and Jian-Wei Pan ${ }^{1,2 \uparrow}$
}

Scalable quantum-information processing requires the capability of storing quantum states ${ }^{1,2}$. In particular, a longlived storable and retrievable quantum memory for single excitations is of key importance to long-distance quantum communication with atomic ensembles and linear optics ${ }^{3-7}$. Although atomic memories for classical light ${ }^{8}$ and continuous variables ${ }^{9}$ have been demonstrated with millisecond storage time, lifetimes of only around $10 \mu \mathrm{s}$ have been reported for quantum memories storing single excitations ${ }^{10-13}$. Here we present an experimental investigation into extending the storage time of quantum memory for single excitations. We identify and isolate distinct mechanisms responsible for the decoherence of spin waves in atomic-ensemble-based quantum memories. By exploiting magnetic-field-insensitive statesso-called clock states-and generating a long-wavelength spin wave to suppress dephasing, we succeed in extending the storage time of the quantum memory to $1 \mathrm{~ms}$. Our result represents an important advance towards long-distance quantum communication and should provide a realistic approach to large-scale quantum information processing.

The quantum repeater with atomic ensembles and linear optics has attracted broad interest in recent years, as it holds promise to implement long-distance quantum communication and the distribution of entanglement over quantum networks. Following the protocol proposed in ref. 3 and the subsequent improved schemes ${ }^{4-7}$, significant experimental progress has been accomplished, including the coherent manipulation of the stored excitation in one $e^{10,11}$ or two ${ }^{14-16}$ atomic ensembles, the demonstration of memory-built-in quantum teleportation ${ }^{17}$ and the realization of a building block of the quantum repeater ${ }^{13,18}$. In these experiments, the atomic ensembles serve as the storable and retrievable quantum memory for single excitations.

Despite the advances achieved in manipulating atomic ensembles, long-distance quantum communication with atomic ensembles remains challenging owing to the short storage time of the quantum memory for single excitations. For example, for direct generation of entanglement between two memory qubits over a few hundred kilometres, we need a memory with a storage time of a few hundred microseconds. However, the longest storage time reported so far is of the order of only $10 \mu$ s (refs 10-13).

It has long been believed that the short coherence time is mainly caused by the residual magnetic field ${ }^{19,20}$. Thereby, storing the collective state in the superposition of the first-order magnetic-field-insensitive states ${ }^{21}$, that is, the 'clock states', is suggested to inhibit this decoherence mechanism ${ }^{19}$. A numerical calculation shows that the expected lifetime is of the order of seconds in this case.

Here we report on our investigation of prolonging the storage time of the quantum memory for single excitations. In the experiment, we find that using only the 'clock state' is not sufficient to obtain the expected long storage time. We further analyse, isolate and identify the distinct decoherence mechanisms, and thoroughly investigate the dephasing of the spin wave (SW) by varying its wavelength. We find that the dephasing of the SW is extremely sensitive to the angle between the write beam and detection mode, especially for small angles. On the basis of this finding, by exploiting the 'clock state' and increasing the wavelength of the SW to suppress the dephasing, we succeed in extending the storage time from $10 \mu \mathrm{s}$ to $1 \mathrm{~ms}$.

The illustration of our experiment is depicted in Fig. 1a,b. A cold ${ }^{87} \mathrm{Rb}$ atomic ensemble released from a magneto-optical trap at a temperature of about $100 \mu \mathrm{K}$ serves as the quantum memory. The two ground states $|g\rangle$ and $|s\rangle$, together with the excited state $|e\rangle$, form a $\Lambda$-type system. A bias magnetic field of about $3.2 \mathrm{G}$ is applied along the axial direction to define the quantization axis. Note that there are three pairs of 'clock states' for the ground states of the ${ }^{87} \mathrm{Rb}$ atom, that is, $(|1,1\rangle,|2,-1\rangle),(|1,0\rangle,|2,0\rangle)$ and $(|1,-1\rangle,|2,1\rangle)$, where we have denoted $|i, j\rangle=\left|5 S_{1 / 2}, F=i, m_{F}=j\right\rangle$. On a timescale of milliseconds, we can use any of them to store the collective excitation, because the decoherence of the 'clock states' caused by magnetic field gradience is negligible. In our experiment, we prepare the atoms in $|1,0\rangle$ to exploit the clock state $(|g\rangle=|1,0\rangle,|s\rangle=|2,0\rangle)$. An off-resonant $\sigma^{-}$-polarized write pulse with wavevector $\mathbf{k}_{\mathrm{W}}$ is applied to the atomic ensemble along the axial direction, inducing spontaneous Raman scattering. The Stokes photon with $\sigma^{-}$polarization and wavevector $\mathbf{k}_{\mathrm{S}}$ is collected at an angle of $\theta=3^{\circ}$ relative to the write beam, as in most of the previous experiments $^{10,13-16,20,22}$. The beam waist of the detection mode is about $100 \mu \mathrm{m}$ in the atomic ensemble. Conditional on detecting a Stokes photon, a collective excited state or an SW is imprinted in the atomic ensemble $e^{3}$, described by

$$
|\psi\rangle=\frac{1}{\sqrt{N}} \sum_{j} \mathrm{e}^{i \Delta \mathbf{k} \cdot \mathbf{r}_{j}}\left|g \ldots s_{j} \ldots g\right\rangle,
$$

with $N$ the number of atoms, $\Delta \mathbf{k}=\mathbf{k}_{\mathrm{W}}-\mathbf{k}_{\mathrm{S}}$ the wavevector of the SW and $\mathbf{r}_{j}$ the coordinate of the $j$ th atom that is excited to $|s\rangle$, denoted by $s_{j}$. After a controllable delay $\delta t$, a strong $\sigma^{+}$-polarized read pulse, counter-propagating with the write light, converts the collective excitation into an anti-Stokes photon, which is $\sigma^{+}$

\footnotetext{
${ }^{1}$ Physikalisches Institut, Universität Heidelberg, Philosophenweg 12, D-69120 Heidelberg, Germany, ${ }^{2}$ Hefei National Laboratory for Physical Sciences at Microscale and Department of Modern Physics, University of Science and Technology of China, Hefei, Anhui 230026, People's Republic of China,

${ }^{3}$ Atominstitut der Österreichischen Universitäten, TU-Wien, A-1020 Vienna, Austria. *These authors contributed equally to this work.

†e-mail:yuaochen@ustc.edu.cn; jian-wei.pan@physi.uni-heidelberg.de.
} 
a

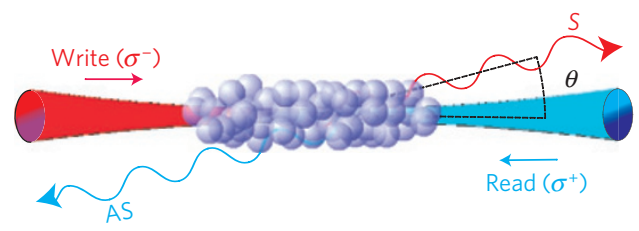

b

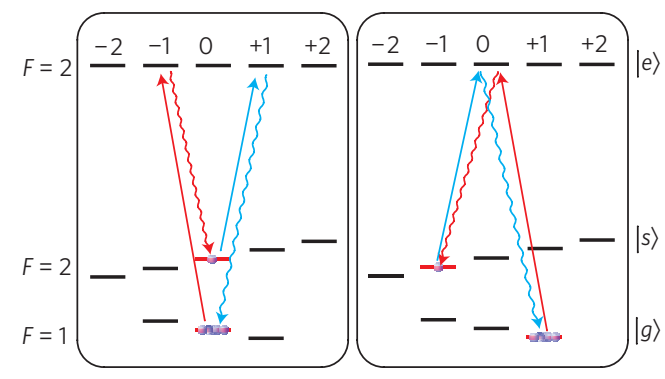

C
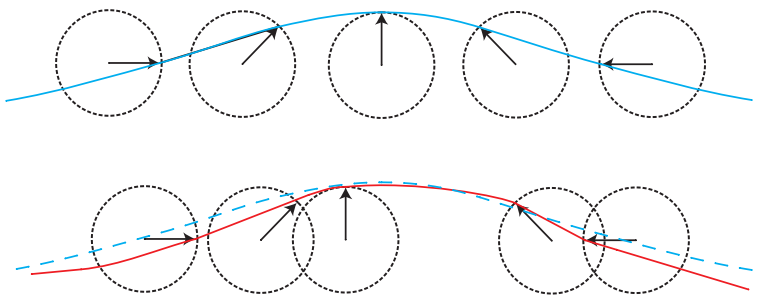

d

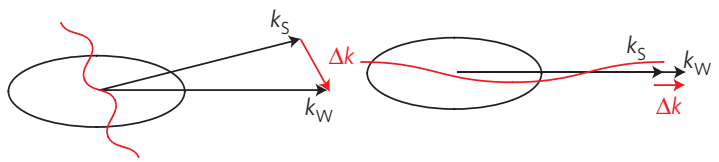

Figure 1 | Schematic view of the experiment. $\mathbf{a}$, The atoms are initially prepared in $|g\rangle$. A weak $\sigma^{-}$-polarized write pulse with a detuning of $\Delta=20 \mathrm{MHz}$ is applied to generate the SW and Stokes photon through spontaneous Raman transition $|g\rangle \rightarrow|e\rangle \rightarrow|s\rangle$. The Stokes photons are detected at an angle of $\theta$ relative to the write beam. After a controllable delay, a strong $\sigma^{+}$-polarized read light with a detuning of $\Delta^{\prime}=6 \mathrm{MHz}$ induces the transition $|s\rangle \rightarrow|e\rangle \rightarrow|g\rangle$, converting the SW into an anti-Stokes photon. $\mathbf{b}$, The structure of atomic transitions $\left({ }^{87} \mathrm{Rb}\right)$ under a weak magnetic field. The left panel corresponds to the experiment with $(|1,0\rangle,|2,0\rangle)$. The right one corresponds to the experiment with $(|1,1\rangle,|2,-1\rangle)$. The photons emitted in undesired transitions are filtered by a polarization filter and a filter cell. c, Illustration of the SW dephasing induced by atomic random motion. The blue curve represents the SW initially stored in the quantum memory. The atoms randomly move along the wavevector direction, resulting in a phase fluctuation. The perturbed SW is represented by the red curve. $\mathbf{d}$, The wavelength of the SW can be controlled by changing the detection configuration. In the collinear case, we have the maximum wavelength.

polarized and spatially mode matched to the Stokes photon directed in the opposite direction. The Stokes (anti-Stokes) photon and the write (read) light are spatially separated.

The quality of the quantum memory can be well characterized by the cross-correlation $g_{\mathrm{S}, \mathrm{AS}}=p_{\mathrm{S}, \mathrm{AS}} /\left(p_{\mathrm{S}} \cdot p_{\mathrm{AS}}\right)$ (see Methods section), with $p_{\mathrm{S}}\left(p_{\mathrm{AS}}\right)$ the probability of detecting a Stokes (anti-Stokes) photon and $p_{S, A S}$ the coincident probability between the Stokes and anti-Stokes channels. The larger the cross-correlation is, the higherquality single-photon source ${ }^{10,11}$ or atom-photon entanglement

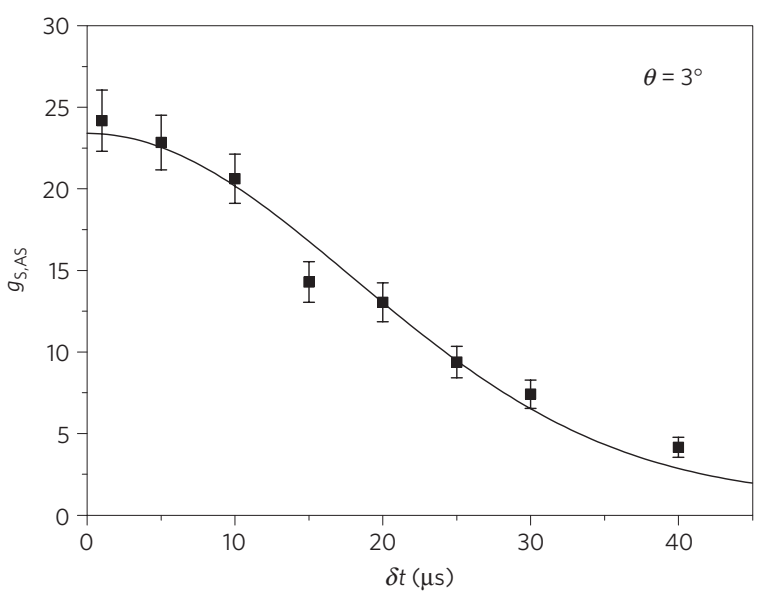

Figure 2 | Measured cross-correlation as a function of the storage time. The cross-correlation $g_{\mathrm{S} \text {, AS }}$ is measured versus the storage time $\delta t$ for $(|1,0\rangle,|2,0\rangle)$ at $\theta=3^{\circ}$. The data are fitted by using $g \mathrm{~S}, \mathrm{AS}(\delta t)=1+C \exp$ $\left(-\delta t^{2} / \tau_{\mathrm{D}}^{2}\right)$. Our data give a lifetime of $\tau_{\mathrm{D}}=(25 \pm 1) \mu \mathrm{s}$. Error bars represent \pm 1 standard deviation.

source ${ }^{18,22,23}$ we can acquire. In the experiment, to evaluate the coherence time of the quantum memory, we measure the crosscorrelation as a function of the time delay, described by ${ }^{11}$ (see the Methods section)

$$
g_{\mathrm{S}, \mathrm{AS}}(\delta t)=1+C \gamma(\delta t)
$$

with $C$ a fitting parameter and $\gamma(\delta t)$ the time-dependent retrieval efficiency. Note that $g_{\mathrm{S}, \mathrm{AS}}>2$ means that the Stokes and anti-Stokes photons are non-classically correlated ${ }^{19,24}$.

The experimental result is shown in Fig. 2. Our data show that the lifetime is longer than our previous results ${ }^{11}$, in which the lifetime is mainly limited by the residual magnetic field ${ }^{19}$, but far from the theoretical predication for the 'clock state'. We carefully analyse the decoherence mechanism of the quantum memory and find that the short lifetime can be explained by the dephasing of the SW induced by atomic random motion ${ }^{12,25}$. This decoherence mechanism plays an important role in many previous experiments ${ }^{10,13-16,20,22}$, but it has not attracted sufficient attention.

The dephasing can be understood as follows. As shown in Fig. 1c, assume an SW is stored in the atomic ensemble and will be retrieved after a time delay $\delta t$. During this interval, each atom randomly moves from one point to another. The internal states or the spins of the atoms are conserved because collisions can be safely ignored at low temperature and density. However, the atomic motion leads to a perturbation on the phase of the SW. Consequently, the projection of the perturbed SW on the original one gradually decreases as the delay of the retrieval is increased. In other words, the atomic random motion leads to a random phase fluctuation in the SW and thus causes decoherence. The timescale of the dephasing can be estimated by calculating the average time for the atoms needed to cross $1 / 2 \pi$ of the wavelength of the SW, giving a lifetime of $\tau_{\mathrm{D}} \sim\left(\lambda / 2 \pi v_{\mathrm{S}}\right)$, with $v_{\mathrm{S}}=\sqrt{k_{\mathrm{B}} T / m}$ the one-dimensional average speed, where $k_{\mathrm{B}}$ is the Boltzmann constant, $T$ the average temperature of the atoms, and $\lambda=(2 \pi / \Delta k)$ the wavelength of the SW. A more detailed calculation yields $\gamma(\delta t) \sim \mathrm{e}^{-\delta t^{2} / \tau_{\mathrm{D}}^{2}}$, with a lifetime $\tau_{\mathrm{D}}=\left(1 / \Delta k v_{\mathrm{S}}\right)$ (see the Methods section). In our case, the angle $\theta$ between $\mathbf{k}_{\mathrm{W}}$ and $\mathbf{k}_{\mathrm{S}}$ determines the wavelength of the SW according to $\Delta k=\left|\mathbf{k}_{\mathrm{W}}-\mathbf{k}_{\mathrm{S}}\right| \simeq k_{\mathrm{W}} \sin \theta$. For $\theta=3^{\circ}$, a simple calculation gives $\lambda=15 \mu \mathrm{m}$ and then $\tau_{\mathrm{D}}=25 \mu \mathrm{s}$. By fitting the data in Fig. 2 with $g_{\mathrm{S}, \mathrm{AS}}(\delta t)=1+C \exp \left(-\delta t^{2} / \tau_{\mathrm{D}}^{2}\right)$, we obtain a lifetime of $\tau_{\mathrm{D}}=(25 \pm 1) \mu \mathrm{s}$, which is consistent with the theoretical calculation. Note that, besides the atomic random motion, the 

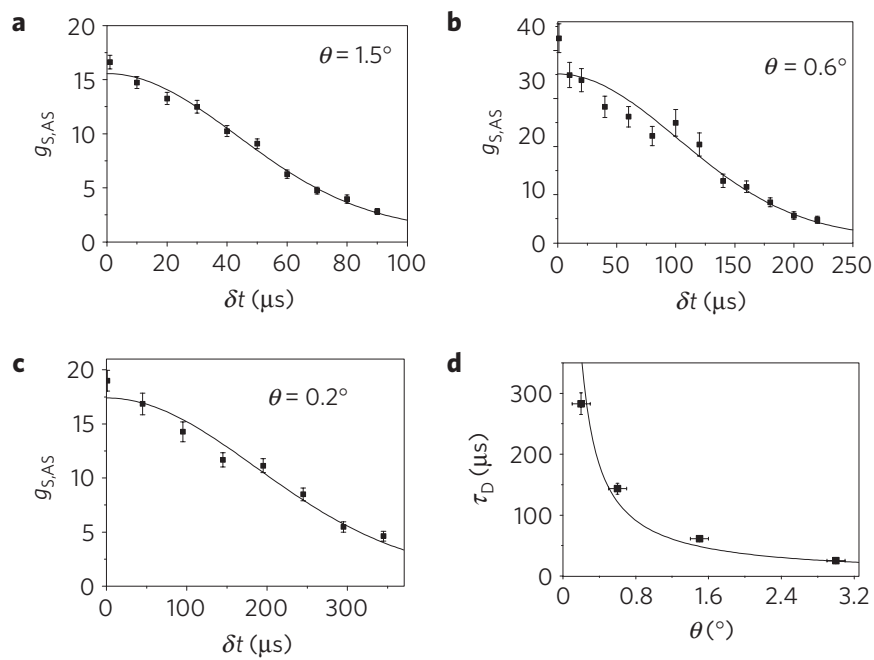

Figure 3 | Lifetime measurement results at different angles. a-c, The cross-correlation $g_{\mathrm{S}, \mathrm{AS}}$ versus the storage time $\delta t$ for different angles. $\mathbf{a , b},(|1,0\rangle,|2,0\rangle)$ at $\theta=1.5^{\circ}$ and $0.6^{\circ}$, respectively. The data are fitted by using $g_{\mathrm{S}, \mathrm{AS}}(\delta t)=1+\mathrm{C} \exp \left(-\delta t^{2} / \tau_{\mathrm{D}}^{2}\right) \cdot \mathbf{c},(|1,1\rangle,|2,-1\rangle)$ at $\theta=0.2^{\circ}$. In this case we take into account the effect of loss of atoms and fit the data by using $g_{\mathrm{S}, \mathrm{AS}}(\delta t)=1+\operatorname{Cexp}\left(-\delta t^{2} / \tau_{\mathrm{D}}^{2}\right) /\left(1+A \delta t^{2}\right)$, with $A$ the fitting parameter obtained from the collinear configuration. The fitted lifetime for each case is $(\mathbf{a}) \tau_{\mathrm{D}}=(61 \pm 2) \mu \mathrm{s}$, (b) $\tau_{\mathrm{D}}=(144 \pm 9) \mu \mathrm{s}$,

(c) $\tau_{\mathrm{D}}=(283 \pm 18) \mu \mathrm{s}$. By reducing the angle, the lifetime is increased from 25 to $283 \mu \mathrm{s}$, which implies that the decoherence is mainly caused by the dephasing induced by atomic random motion. $\mathbf{d}$, The measured lifetime $\tau_{\mathrm{D}}$ as a function of detection angle $\theta$, where the horizontal error bars indicate measurement errors in the angles. The solid line is the theoretical curve with $T=100 \mu \mathrm{K}$. The experimental results are in good agreement with the theoretical predications. The error bars indicate \pm 1 standard deviation.

collisions between atoms may also affect the phase of the SW. However, the collision rate can be estimated by $\Gamma \sim n v_{\mathrm{s}} \sigma \sim 1 \mathrm{~Hz}$, where the atomic density $n=10^{10} \mathrm{~cm}^{-3}$ and the $s$-wave scattering cross-section $\sigma=8 \pi a^{2}$ with the scattering length $a=6 \mathrm{~nm}$. Thus, on the timescale of milliseconds, the collisions can be safely ignored.

To further confirm that the decoherence is mainly caused by the dephasing induced by atomic motion, we increase the wavelength of the SW by decreasing the detection angle (see Fig. 1d). In this way, according to the above model, the dephasing is suppressed and the storage time will be extended. In our experiment, we select angles of $\theta=1.5^{\circ}, 0.6^{\circ}$ and $0.2^{\circ}$ and measure the lifetime of the quantum memory for each configuration. Note that for $\theta=0.2^{\circ}$ the write (read) and Stokes (anti-Stokes) lights with the same polarization cannot be spatially separated, and therefore we use another pair of 'clock states' $(|g\rangle=|1,1\rangle,|s\rangle=|2,-1\rangle)$ by preparing the atoms in $|1,1\rangle$. In this case, the Stokes (anti-Stokes) photon is $\sigma^{+}\left(\sigma^{-}\right)$ polarized and thus can be separated by a Glan-laser prism from the write (read) pulse.

The experimental results are shown in Fig. 3a-c. As expected, the dephasing of the SW dominates. The lifetime increases from 25 to $283 \mu$ s when reducing the angle owing to the extended wavelength of the SW. Our results clearly show that the dephasing of the SW is extremely sensitive to the small angle between the write beam and Stokes modes, and that the long-wavelength SW is robust against the dephasing induced by atomic random motion. Note that, for $\theta=0.2^{\circ}$, the data are fitted taking into account the effect of loss of atoms. The measured lifetime $\tau_{\mathrm{D}}$ is shown in Fig. $3 \mathrm{~d}$ as a function of angle $\theta$. The solid line is the theoretical curve $\tau_{\mathrm{D}}=\left(1 / \Delta k v_{\mathrm{s}}\right)$, with $v_{\mathrm{s}}=0.1 \mathrm{~m} \mathrm{~s}^{-1}$ corresponding to a temperature of $T \simeq 100 \mu \mathrm{K}$. The good agreement between theory and experiment implies that our work provides an alternative approach to measure the temperature

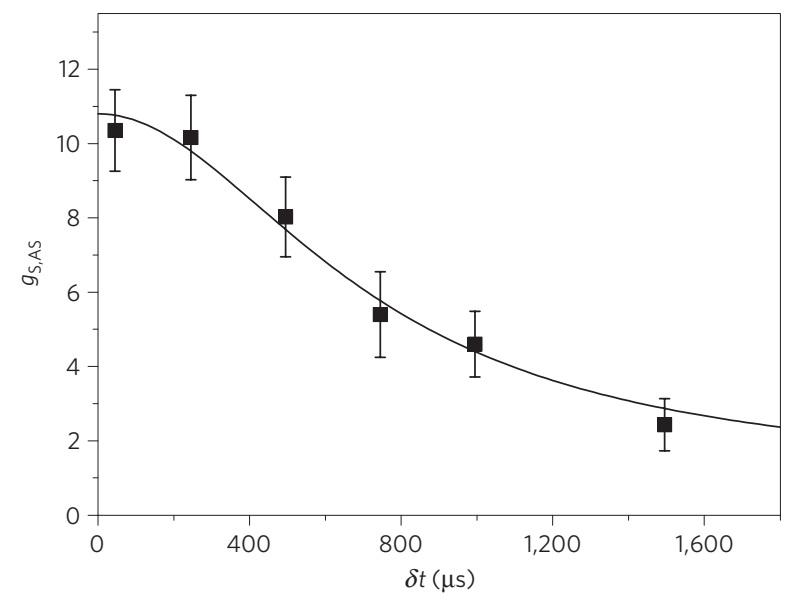

Figure 4 | Lifetime at collinear configuration. The cross-correlation $g_{\mathrm{S}, \mathrm{AS}}$ versus the storage time $\delta t$ for $(|1,1\rangle,|2,-1\rangle)$ at $\theta=0^{\circ}$, which enables a maximal-wavelength $\mathrm{SW}$. The data are fitted by using $g_{\mathrm{S}, \mathrm{AS}}(\delta t)=1+\left(\mathrm{C} / 1+A \delta t^{2}\right)$, with $A$ the fitting parameter. Our data give a lifetime of $\tau_{\mathrm{L}}=(1.0 \pm 0.1) \mathrm{ms}$, when the retrieval efficiency $\gamma(\delta t)=\left(1 / 1+A \delta t^{2}\right)$ has dropped to $1 /$ e. Error bars represent \pm 1 standard deviation.

of an atomic ensemble. Moreover, as the lifetime is sensitive only to the velocity of the atoms in the interaction region, which is determined by the waist of the detection mode and is controllable, our method can also be used to measure the velocity distribution of the atomic ensemble by making measurements in different regions.

To further suppress the dephasing and achieve a longer storage time, we use the collinear configuration $\left(\theta=0^{\circ}\right)$, where we have the maximum wavelength of the $\mathrm{SW}$ of $\lambda \simeq 4.4 \mathrm{~cm}$, which yields a computed lifetime of $\tau_{\mathrm{D}}=72 \mathrm{~ms}$. In this case, the decoherence due to the loss of atoms, which causes a typical lifetime of a few hundred microseconds, is isolated as the principal source of decoherence. This mechanism can be estimated by calculating the average time for the atoms leaving the pencil shaped interaction region, where the thermal motion in the radial direction dominates. At temperature $T$, an atomic cloud with a cross-section radius $r_{0}$ expands according to $r^{2}(\delta t)=r_{0}^{2}+v_{r}^{2} \delta t^{2}$, with the average speed in the radial direction $v_{r}=\sqrt{2 k_{\mathrm{B}} T / m}$. The retrieval efficiency can be given by $\gamma(\delta t)=r_{0}^{2} / r^{2}(\delta t)=1 /\left(1+v_{r}^{2} \delta t^{2} / r_{0}^{2}\right)$. Thereby, when $\gamma\left(\tau_{\mathrm{L}}\right)=1 / \mathrm{e}$, only a fraction $1 / \mathrm{e}$ of the atoms remain in the interaction region, giving a lifetime of $\tau_{\mathrm{L}} \simeq\left(1.31 r_{0} / v_{r}\right)$. For $r_{0}=100 \mu \mathrm{m}$ as the waist of the detection mode and $T=100 \mu \mathrm{K}$, a direct calculation yields $\tau_{\mathrm{L}}=950 \mu \mathrm{s}$, which is much smaller than the dephasing time due to atomic motion and decoherence induced by the magnetic field. The experimental result is shown in Fig. 4, where the 'clock states' $(|1,1\rangle,|2,-1\rangle)$ are used as well. Our data show a lifetime of $\tau_{\mathrm{L}}=(1.0 \pm 0.1) \mathrm{ms}$, when the retrieval efficiency has dropped to $1 / \mathrm{e}$. The experimental result is in good agreement with the theoretical estimate.

In our experiment, we have isolated and identified different decoherence mechanisms of the quantum memory for single excitations and thoroughly investigated the dephasing of the stored SW by varying its wavelength. Moreover, we have successfully realized a long-lived quantum memory for single collective excitation with the storage time of $1 \mathrm{~ms}$, which is 30 times longer than the best result reported so far ${ }^{10}$, and is long enough for photon transmission over 100 kilometres. In our experiment, the coherence time of the quantum memory is limited by the decoherence due to loss of atoms, which can be suppressed by lowering the temperature through optical molasses. A storage time of $3 \mathrm{~ms}$ is achievable by reducing the temperature to $10 \mu \mathrm{K}$. This will be the upper limit for an atomic memory with a magneto-optical trap, because a 
longer storage time is prohibited by the free fall of the atoms under gravity. Further improvement might be achieved by trapping the atoms in an optical dipole trap ${ }^{19,26}$. In this case, the principal decoherence mechanism is the diffusion caused by collisions, which will give a lifetime of a few tens of milliseconds. To inhibit the collision-induced diffusion, we have to trap the atoms in a deep optical lattice $^{27}$ or use solid-state systems ${ }^{28}$, where each atom is tightly confined on a single site. The optical lattice has the potential to store the collective excitation for a few tens of seconds, which is required for a robust and efficient quantum repeater with atomic ensembles ${ }^{29}$. The idea presented in this work can also be applied to quantum memory based on electromagnetically induced transparency ${ }^{20,30,31}$.

Our work opens up an exciting possibility to implement many tasks of quantum information processing. Combined with the techniques developed in recent years, we can implement a high-quality on-demand single-photon source, deterministic preparation of multiqubit entanglement, generation of entanglement between two remote atomic memory qubits over a few hundred kilometres and even construction of long-lived quantum nodes for a quantum repeater. Besides, our work also provides an approach to measure the temperature or the velocity distribution of an atomic ensemble. Furthermore, as the decoherence of the SW is controllable, we can measure certain important physical quantities by introducing additional physical mechanisms. For example, when performing experiments in an optical dipole trap, the lifetime is determined by collision between atoms ${ }^{25}$. Thereby, the $s$-wave scattering cross-section or scattering length might be measured using our approach.

\section{Methods}

Dephasing of spin wave induced by atomic random motion. Assume the $j$ th atom moves to $\mathbf{r}_{j}(\delta t)=\mathbf{r}_{j}+\mathbf{v}_{j} \delta t$ after a storage time of $\delta t$. The collective state or SW freely evolves to

$$
\left|\psi_{\mathrm{D}}\right\rangle=\frac{1}{\sqrt{N}} \sum_{j} \mathrm{e}^{i \Delta \mathbf{k} \cdot \mathbf{r}_{j}(\delta t)}\left|g \ldots s_{j} \ldots g\right\rangle,
$$

where we have ignored the effect of magnetic field for simplicity. The retrieval efficiency is proportional to the overlap between the original SW and the perturbed one,

$$
\gamma(\delta t) \sim\left|\left\langle\psi \mid \psi_{\mathrm{D}}\right\rangle\right|^{2}=\left|\frac{1}{N} \sum_{j} \mathrm{e}^{i \Delta \mathbf{k} \cdot v_{j} \delta t}\right|^{2}=\left|\int f(v) \mathrm{e}^{i \Delta \mathbf{k} \cdot v_{j} \delta t} \mathrm{~d} v\right|^{2},
$$

with $f(\mathbf{v})$ the velocity distribution. Assume $f(v) \sim \mathrm{e}^{-m v^{2} / 2 k_{\mathrm{B}} T}$ is a Boltzmann distribution at temperature $T$. Integrating over all possible velocities, we obtain $\gamma(\delta t) \sim \mathrm{e}^{-\delta t^{2} / \tau_{\mathrm{D}}^{2}}$, with the lifetime $\tau_{\mathrm{D}}=\left(1 / \Delta k v_{\mathrm{s}}\right)$. Note that the retrieval efficiency also depends on other factors, for example the optical depth, the coupling strength and so on.

Cross-correlation function. The quality of the quantum memory can be well characterized by the cross-correlation $g_{\mathrm{S}, \mathrm{AS}}=p_{\mathrm{S}, \mathrm{AS}} /\left(p_{\mathrm{S}} \cdot p_{\mathrm{AS}}\right)$. For $g_{\mathrm{S}, \mathrm{AS}} \gg 1$, when the atomic ensemble is used as a single-photon source ${ }^{10,11}$, the autocorrelation of the heralded single photons can be estimated by $\alpha \simeq 4 /\left(g_{\mathrm{S}, \mathrm{AS}}-1\right)$. When the atomic ensemble is exploited to prepare an atom-photon entanglement ${ }^{18,22,23}$, the violation of the Clause-Horne-Shimony-Holt-type Bell inequality, which is insensitive to the relative phase between the vacuum and the single excitation, can be approximated by $S \simeq 2 \sqrt{2}\left(g_{\mathrm{S}, \mathrm{AS}}-1\right) /\left(g_{\mathrm{S}, \mathrm{AS}}+1\right)$. Thereby, a large cross-correlation function indicates a high-quality quantum memory. The non-classical correlation between Stokes and anti-Stokes fields is characterized by the violation of Cauchy-Schwarz inequality $g_{\mathrm{S}, \mathrm{AS}}^{2} \leqslant g_{\mathrm{S}, \mathrm{S}} g_{\mathrm{AS}, \mathrm{AS}}$, with $g_{\mathrm{S}, \mathrm{S}}$ and $g_{\mathrm{AS}, \mathrm{AS}}$ the autocorrelations of Stokes and anti-Stokes fields. In the ideal case $g_{\mathrm{S}, \mathrm{S}}=g_{\mathrm{AS}, \mathrm{AS}}=2$, but in practice they are usually smaller than 2 owing to the background noise ${ }^{24}$. Thereby, we can infer that when $g_{\text {S.AS }}>2$ the Stokes and anti-Stokes field are non-classically correlated ${ }^{19}$ In the experiment, we measure the decay of the cross-correlation to evaluate the lifetime of the quantum memory. By ignoring the noise in the Stokes channel, we have ${ }^{11} p_{\mathrm{S}}=\chi \eta_{\mathrm{S}}, p_{\mathrm{AS}}=\chi \gamma(\delta t) \eta_{\mathrm{AS}}+B \eta_{\mathrm{AS}}$ and $p_{\mathrm{S}, \mathrm{AS}}=\chi \gamma(\delta t) \eta_{\mathrm{S}} \eta_{\mathrm{AS}}+p_{\mathrm{S}} p_{\mathrm{AS}}$, with $\chi$ the excitation probability, $\gamma(\delta t)$ the time-dependent retrieval efficiency, $\eta_{\mathrm{S}}\left(\eta_{\mathrm{AS}}\right)$ the overall detection efficiencies in the Stokes (anti-Stokes) channels and $B$ the background noise in the anti-Stokes channel. Thereby the decay of the cross-correlation function can be approximated by

$$
g_{\mathrm{S}, \mathrm{AS}}(\delta t)=1+\frac{\gamma(\delta t)}{\chi \gamma(\delta t)+B} \simeq 1+C \gamma(\delta t),
$$

where $C$ is a fitting parameter. In our experiment, $p_{\mathrm{S}} \sim 0.003$ and the cross-correlation is comparable with previous works ${ }^{11}$. For small angles and in the collinear regime, the cross-correlation is lower because of the relatively large background noise due to the write and read beams. Note that by reducing the power and waist of write and read beams, and improving the filtering techniques, a high cross-correlation of about 100 can be achieved in the collinear configuration ${ }^{11}$.

Experimental details. In the experiment, more than $10^{8}{ }^{87} \mathrm{Rb}$ atoms are collected by the magneto-optical trap with an optical depth of about 5 and a temperature of about $100 \mu \mathrm{K}$ within $20 \mathrm{~ms}$ at a repetition rate of $40 \mathrm{~Hz}$. The trapping magnetic field and repumping beams are then quickly switched off. After $0.5 \mathrm{~ms}$, the bias magnetic field is switched on, whereas the cooler beams stay on for another $0.5 \mathrm{~ms}$ before being switched off in order to prepare the atoms in the $\left|5 S_{1 / 2}, F=1\right\rangle$ ground state. Then, within another $4 \mathrm{~ms}$, experimental trials (each consisting of pumping, write and read pulses) are repeated with a controllable period depending on the desired retrieval time. To optically pump the atoms to the desired sublevel, we switch on two pumping beams in each experimental trial before the write and read process: one couples all the dipole transitions of $\left|5 S_{1 / 2}, F=2\right\rangle \rightarrow\left|5 P_{3 / 2}, F^{\prime}=2\right\rangle$ for $2 \mu \mathrm{s}$, and the other couples the $\pi\left(\sigma^{+}\right)$transition of $\left|5 S_{1 / 2}, F=1\right\rangle \rightarrow\left|5 P_{1 / 2}, F^{\prime}=1\right\rangle$ for $|1,0\rangle(|1,1\rangle)$ for $1.7 \mu$ s. From the experimental result, we estimate that more than $80 \%$ of the atoms are prepared in the desired state.

For $\theta=3^{\circ}, 1.5^{\circ}$ and $0.6^{\circ}$, the Stokes (anti-Stokes) photon and the write (read) light can be spatially separated and thus we can choose any of the three pairs of 'clock states'. Because the retrieval efficiency is proportional to the coupling strength of the transition $|e\rangle \rightarrow|g\rangle$, we choose the clock state $(|1,0\rangle,|2,0\rangle)$ to obtain higher retrieval efficiency, whereas for smaller angles $\theta=0.2^{\circ}$ and $0^{\circ}$, the two beams with the same polarization cannot be spatially separated, and thereby we have to use the other two 'clock states'. In this case we choose the 'clock state' $(|1,1\rangle,|2,-1\rangle)$, because the energy level $|1,1\rangle$ is lower than $|1,-1\rangle$ under the bias magnetic field and the pumping effect is better. The overall retrieval efficiencies, including the transmission efficiency of the filters and optical components, the coupling efficiency of the fibre coupler and the detector quantum efficiency, are about $2 \%$ for $(|1,0\rangle,|2,0\rangle)$ at $\theta=3^{\circ}, 1.5^{\circ}$, and $0.6^{\circ}, 1 \%$ for $(|1,1\rangle,|2,-1\rangle)$ at $\theta=0.2^{\circ}$ and $0.8 \%$ for $(|1,1\rangle,|2,-1\rangle)$ at $\theta=0^{\circ}$. The retrieval efficiency at $\theta=0^{\circ}$ is a little lower than that at $\theta=0.2^{\circ}$ because one more etalon was used to filter the excitation beams. These correspond to $15 \%$ and $10 \%$ of the original retrieval efficiency for $(|1,0\rangle,|2,0\rangle)$ and $(|1,1\rangle,|2,-1\rangle)$, respectively. The low overall retrieval efficiency is caused by the transmission loss, the mode mismatch, the imperfect pumping and the imperfect polarization of the write and read light.

\section{Received 27 May 2008; accepted 7 November 2008; published online 7 December 2008}

\section{References}

1. Briegel, H. J., Dür, W., Cirac, J. I. \& Zoller, P. Quantum repeaters: The role of imperfect local operations in quantum communication. Phys. Rev. Lett. 81, 5932-5935 (1998).

2. Knill, E., Laflamme, R. \& Milburn, G. J. A scheme for efficient quantum computation with linear optics. Nature 409, 46-52 (2001).

3. Duan, L.-M., Lukin, M. D., Cirac, J. I. \& Zoller, P. Long-distance quantum communication with atomic ensembles and linear optics. Nature 414 413-418 (2001).

4. Zhao, B., Chen, Z.-B., Chen, Y.-A., Schmiedmayer, J. \& Pan, J.-W. Robust creation of entanglement between remote memory qubits. Phys. Rev. Lett. 98, 240502 (2007).

5. Chen, Z.-B., Zhao, B., Chen, Y.-A., Schmiedmayer, J. \& Pan, J.-W. Fault-tolerant quantum repeater with atomic ensembles and linear optics. Phys. Rev. A 76, 022329 (2007).

6. Jiang, L., Taylor, J. M. \& Lukin, M. D. Fast and robust approach to long-distance quantum communication with atomic ensembles. Phys. Rev. A 76, 012301 (2007).

7. Collins, O. A., Jenkins, S. D., Kuzmich, A. \& Kennedy, T. A. B. Multiplexed memory insensitive quantum repeaters. Phys. Rev. Lett. 98, 060502 (2007).

8. Liu, C., Dutton, Z., Behroozi, C. H. \& Hau, L. V. Observation of coherent optical information storage in an atomic medium using halted light pulses. Nature 409, 490-493 (2001).

9. Julsgaard, B., Sherson, J., Cirac, J. I., Fiurášek, J. \& Polzik, E. S. Experimental demonstration of quantum memory for light. Nature 432, 482-486 (2004)

10. Matsukevich, D. N. et al. Deterministic single photons via conditional quantum evolution. Phys. Rev. Lett. 97, 013601 (2006) 
11. Chen, S. et al. Deterministic and storable single-photon source based on quantum memory. Phys. Rev. Lett. 97, 173004 (2006).

12. Simon, J., Tanji, H., Thompson, J. K. \& Vuletic, V. Interfacing collective atomic excitations and single photons. Phys. Rev. Lett. 98, 183601 (2007).

13. Chou, C.-W. et al. Functional quantum nodes for entanglement distribution over scalable quantum networks. Science 316, 1316-1320 (2007).

14. Felinto, D. et al. Conditional control of the quantum states of remote atomic memories for quantum networking. Nature Phys. 2, 844-848 (2006).

15. Chanelière, T. et al. Quantum interference of electromagnetic fields from remote quantum memories. Phys. Rev. Lett. 98, 113602 (2007).

16. Yuan, Z.-S. et al. Synchronized independent narrow-band single photons and efficient generation of photonic entanglement. Phys. Rev. Lett. 98, 180503 (2007).

17. Chen, Y.-A. et al. Memory-built-in quantum teleportation with photonic and atomic qubits. Nature Phys. 4, 103-107 (2008).

18. Yuan, Z.-S. et al. Experimental demonstration of a BDCZ quantum repeater node. Nature 454, 1098-1101 (2008).

19. Felinto, D., Chou, C. W., de Riedmatten, H., Polyakov, S. V. \& Kimble, H. J. Control of decoherence in the generation of photon pairs from atomic ensembles. Phys. Rev. A 72, 053809 (2005).

20. Choi, K. S., Deng, H., Laurat, J. \& Kimble, H. J. Mapping photonic entanglement into and out of a quantum memory. Nature 452, 67-71 (2008).

21. Harber, D. M., Lewandowski, H. J., McGuirk, J. M. \& Cornell, E. A. Effect of cold collisions on spin coherence and resonance shifts in a magnetically trapped ultracold gas. Phys. Rev. A 66, 053616 (2002).

22. Chen, S. et al. Demonstration of a stable atom-photon entanglement source for quantum repeaters. Phys. Rev. Lett. 99, 180505 (2007).

23. de Riedmatten, H. et al. Direct measurement of decoherence for entanglement between a photon and stored atomic excitation. Phys. Rev. Lett. 97, 113603 (2006).
24. Chou, C.W., Polyakov, S. V., Kuzmich, A. \& Kimble, H. J. Single-photon generation from stored excitation in an atomic ensemble. Phys. Rev. Lett. 92, 213601 (2004).

25. Mewes, C. \& Fleischhauer, M. Decoherence in collective quantum memories for photons. Phys. Rev. A 72, 022327 (2005).

26. Grimm, R., Weidemüller, M. \& Ovchinnikov, Y. B. Optical dipole traps for neutral atoms. Adv. Atom. Mol. Opt. Phys. 42, 95-170 (2000).

27. Greiner, M., Mandel, O., Esslinger, T., Hänsch, T.W. \& Bloch, I. Quantum phase transition from a superfluid to a Mott insulator in a gas of ultracold atoms. Nature 415, 39-44 (2002).

28. Longdell, J. J., Fraval, E., Sellars, M. J. \& Manson, N. B. Stopped light with storage times greater than one second using electromagnetically induced transparency in a solid. Phys. Rev. Lett. 95, 063601 (2005).

29. Sangouard, N. et al. Robust and efficient quantum repeaters with atomic ensembles and linear optics. Phys. Rev. A 77, 062301 (2008)

30. Fleischhauer, M. \& Lukin, M. D. Dark-state polaritons in electromagnetically induced transparency. Phys. Rev. Lett. 84, 5094-5097 (2000).

31. Chanelière, T. et al. Storage and retrieval of single photons transmitted between remote quantum memories. Nature 438, 833-836 (2005).

\section{Acknowledgements}

We acknowledge M. Fleischhauer and Y. J. Deng for useful discussions. This work was supported by the Deutsche Forschungsgemeinschaft (DFG), the Alexander von Humboldt Foundation, an ERC grant, the National Fundamental Research Program (grant No. 2006CB921900), the CAS and the NNSFC.

\section{Additional information}

Reprints and permissions information is available online at http://npg.nature.com/ reprintsandpermissions. Correspondence and requests for materials should be addressed to Y.-A.C. or J.-W.P. 